Published in final edited form as:

Kumru, B., Antonietti, M., \& Schmidt, B. V. K. J. (2017). Enhanced Dispersibility of Graphitic Carbon Nitride Particles in Aqueous and Organic Media via a One Pot Grafting Approach. Langmuir, 33(38), 9897-9906. doi:10.1021/acs.langmuir.7b02441.

\title{
Enhanced Dispersibility of Graphitic Carbon Nitride Particles in Aqueous and Organic Media via a One Pot Grafting Approach
}

Baris Kumru, Markus Antonietti, Bernhard V.K.J. Schmidt

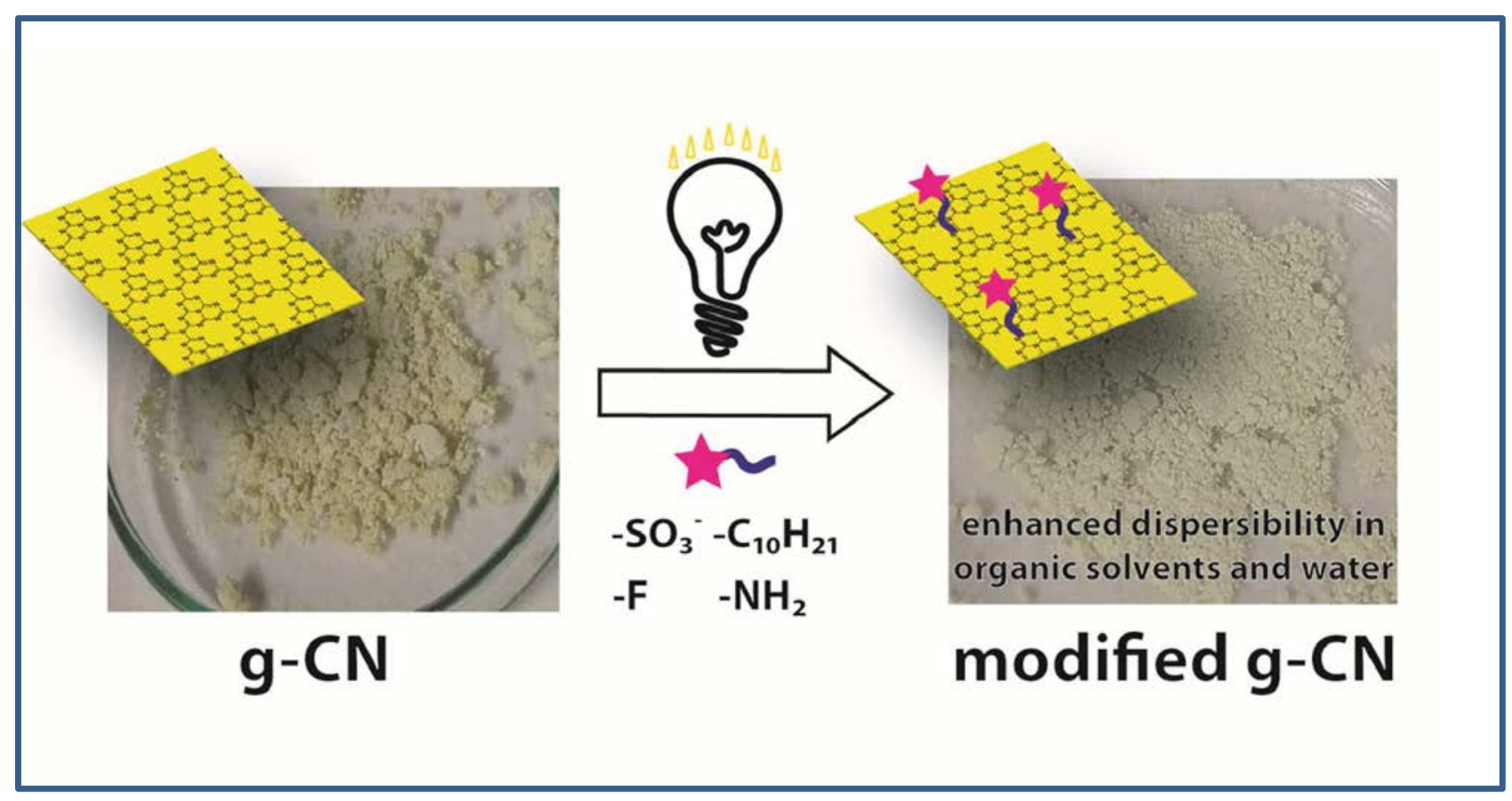




\title{
Enhanced Dispersibility of Graphitic Carbon Nitride
}

\section{Particles in Aqueous and Organic Media via a One}

\section{Pot Grafting Approach}

\author{
Baris Kumru, Markus Antonietti, Bernhard V.K.J. Schmidt* \\ Department of Colloid Chemistry, Max Planck Institute of Colloids and Interfaces, Research \\ Campus Golm, 14424 Potsdam (Germany)
}

KEYWORDS Carbon Nitride, Dispersibility, Grafting

ABSTRACT A facile route to synthesize hydrophilically or hydrophobically grafted graphitic carbon nitride $(\mathrm{g}-\mathrm{CN})$ is reported. For this purpose, functionalized olefinic molecules with low polymerization tendency are utilized for grafting onto the surface in order to preserve the features of g-CN while improving its dispersibility. One pot, visible light induced grafting yields highly dispersible g-CNs either in aqueous or organic media. Moreover, functional groups such as amine can be introduced, which yields $\mathrm{pH}$ dependent dispersibility in aqueous media. Compared with unfunctionalized g-CN low sonication times are sufficient to redisperse g-CN. In addition, due to increased dispersion stability, higher amounts of functionalized g-CN can be dispersed (up to $10 \%$ in aqueous dispersion and $2 \%$ in organic dispersion), when compared to unfunctionalized g-CN. 


\section{Introduction}

Graphitic carbon nitride $(\mathrm{g}-\mathrm{CN})$ is a metal-free photocatalytic material significantly active in the visible light range between $390-480 \mathrm{~nm} .{ }^{1,2}$ It is generally composed of repeating tri-s-triazine rings $^{3}$, and its properties can be adjusted by changing the synthesis conditions, such as polymerization temperature ${ }^{4,5}$ and utilized precursors. ${ }^{6,7}$ As heterogeneous catalyst, it raised interest e.g. in $\mathrm{CO}_{2}$ reduction, ${ }^{8}$ water splitting, ${ }^{9-11}$ designed organic reactions, ${ }^{12}$ photodegradation $^{13,14}$ and very recently as an amphiphile and Pickering emulsion-dispersion stabilizer. ${ }^{15}$ The high chemical and thermal stability of $\mathrm{g}-\mathrm{CN}$ as well as convenient synthesis and low cost precursors make it also a promising candidate for many photocatalytic applications. ${ }^{16}$ Lately, g-CN was investigated as radical initiator in polymerization reactions together with tertiary amines as co-catalyst. ${ }^{17}$ Therein, researchers successfully integrated g-CN as radical initiator in free radical and controlled polymerizations of styrene as well as methyl methacrylate. Moreover, Weber et. al. investigated the formation of polymer-mesoporous g-CN composites by aerosol polymerization using $N$-methyldiethanolamine as co-catalyst. ${ }^{18}$ Recently, our group investigated the role of $\mathrm{g}-\mathrm{CN}$ in the formation of reinforced hydrogels and photocatalytically active hydrogels without any co-catalyst. ${ }^{19,20}$ All these experiments suggested the formation of radicals on the surface of $\mathrm{g}-\mathrm{CN}$, which are capable of initiation of free radical polymerization.

Modification of $\mathrm{g}-\mathrm{CN}$ is relevant when the activity of unmodified $\mathrm{g}-\mathrm{CN}$ is not at the desired level. There are two routes towards modification, either via changing synthesis conditions or post-synthesis modification. $^{21}$ Changing synthesis conditions such as preorganization of monomers, ${ }^{22,23}$ solvent addition ${ }^{24}$ and polymerization temperature ${ }^{4,25}$ yields g-CNs with different specific surface area ${ }^{26}$ and functional groups. ${ }^{27,28}$ Post synthetic methods can be considered as protonation of $\mathrm{g}-\mathrm{CN},{ }^{29}$ doping $^{30}$ and macromolecular grafting. ${ }^{31}$ For heterogeneous catalysis 
systems, formation of active centers and their interaction with the solvent are important factors for the rate of reaction. ${ }^{32}$ These modified $\mathrm{g}$-CNs are used in enhanced visible light photodegradation $^{33}$ and room temperature esterification of fatty acids to yield biodiesels. ${ }^{34}$ However, the main drawback for the utilization of $\mathrm{g}-\mathrm{CN}$ is weak dispersibility since strong van der Waals attractions ( $\pi-\pi$ stacking) of $\mathrm{sp}^{2}$ carbons cause re-agglomeration in solvents. ${ }^{35}$ Therefore, the use of dispersed g-CN is limited, and only limited amounts of g-CN can be dispersed in time consuming processes as reaggregation is a colloidal process which is highly concentration dependent. The main approaches to enhance dispersibility are hydrophilic modification of $\mathrm{g}-\mathrm{CN}^{36}$ or utilization of additives, such as surfactants or strong acids, during the dispersion step. ${ }^{37}$ Yet these additives can be undesirable for aimed reaction conditions and thus decrease the applicability, e.g. surfactants are challenging to remove after the reaction, strong acids can interfere with reactants or with the reaction mechanism. Recently, Wang and coworkers reported the modification of g-CN nanosheets with oxygen plasma treatment in order to introduce hydroxylamine groups to enhance the degree of protonation. ${ }^{38}$ The reaction conditions required long ultrasonication times to yield nanosheets first and then plasma treatment under high pressure and microwave irradiation. Yet, to the best of our knowledge, there are no reports on $\mathrm{g}-\mathrm{CN}$ dispersion in organic media or $\mathrm{pH}$-dependent $\mathrm{g}-\mathrm{CN}$ dispersion in aqueous medium.

Inspired by our recent work on $\mathrm{CN}$ photoinitiation for hydrogel formation, ${ }^{19,20}$ we here attempt to utilize radicals on the $\mathrm{CN}$ surface for modification: radicals are created on the surface of g-CN via visible light, and various functionalities are integrated. In order to suppress polymerization, functional non-propagating allyl compounds were utilized as depicted in Scheme 1. 

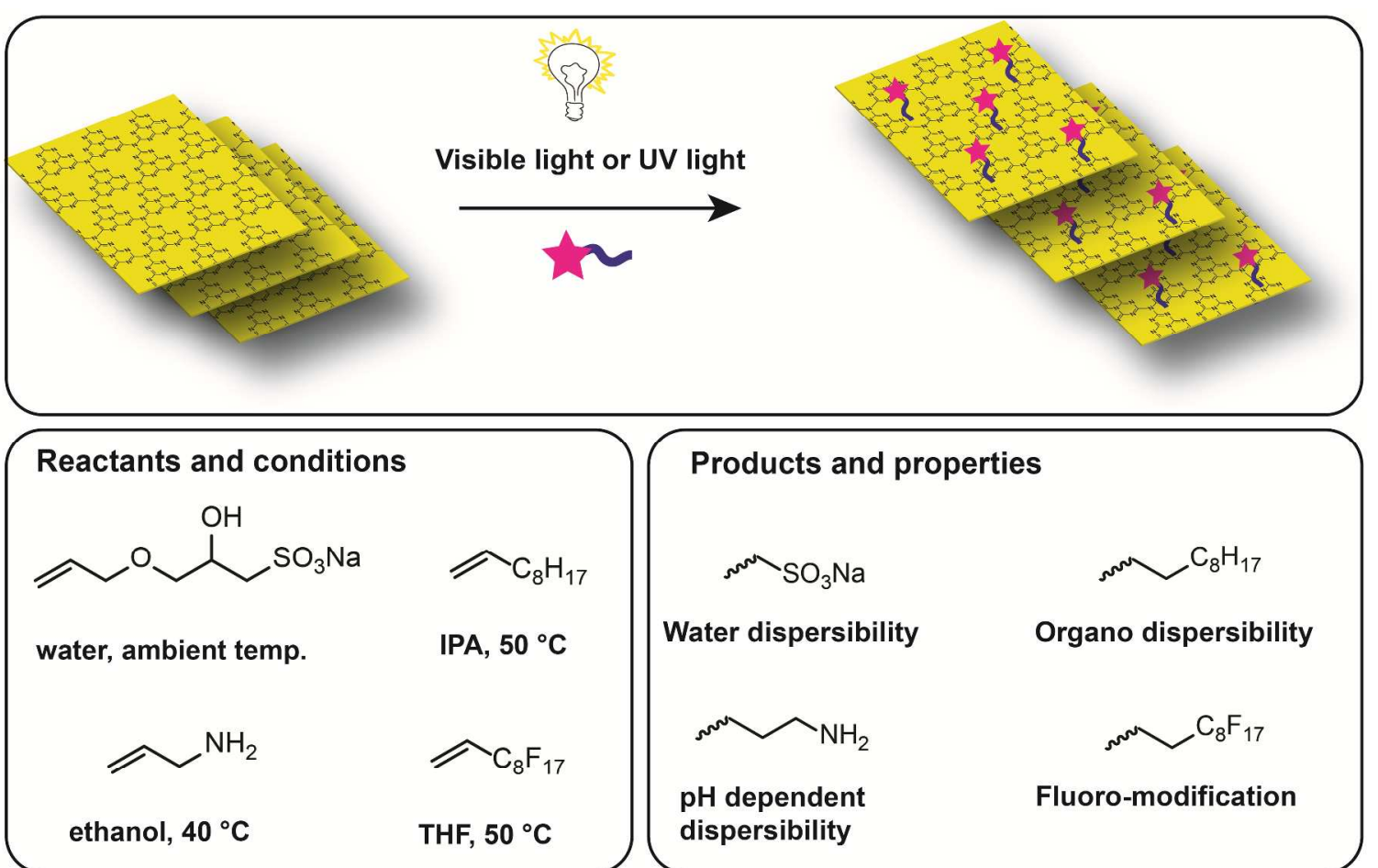

Products and properties

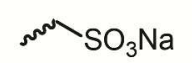

Water dispersibility

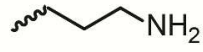

$\mathrm{pH}$ dependent dispersibility

Scheme 1. Overview for g-CN modifications based on light induced grafting. IPA: isopropanol.

Functionalities such as sulfonic acids, alkane chains, amino groups and fluoro groups are introduced to g-CN, which was synthesized from cyanuric acid-melamine (CM) complex and is annotated as CM. Facile light induced syntheses together with a convenient purification process yield highly dispersible g-CNs. It is shown that g-CNs modified with various functionalities enables quick dispersion times and increased solid contents in the respective solvents compared to non-functionalized reference g-CN (up to $10 \mathrm{wt} \%$ in water and $2 \mathrm{wt} . \%$ in acetone). Moreover, it is possible to introduce $\mathrm{pH}$ dependent functionalities via grafting of allylamine.

\section{Experimental Section}

\section{Materials}

All materials were used as purchased unless noted otherwise. Acetone (HPLC grade, Sigma Aldrich), allylamine (98\%, Sigma Aldrich), 3-allyloxy-2-hydroxy-1-propanesulfonic acid 
sodium salt solution (40 wt.\%, AHPA, Sigma Aldrich), cyanuric acid (98\%, Sigma Aldrich), 1decene (94\%, Sigma Aldrich), deuterated chloroform $\left(\mathrm{CDCl}_{3}\right.$, Sigma Aldrich), deuterium oxide ( $\mathrm{D}_{2} \mathrm{O}$, Sigma Aldrich), dichloromethane (DCM, anhydrous 99.8\%, Sigma Aldrich), hexafluorobenzene (99\%, Alfa Aesar), hexane (anhydrous, 95\%, Sigma Aldrich), hydrochloric acid (0.1 N, Alfa Aesar), hydrogen peroxide solution (50 wt.\%, Sigma Aldrich), isopropyl alcohol (IPA, 99.7\%, Sigma Aldrich), melamine (99\%, Sigma Aldrich), 1H,1H,2H-perfluoro-1decene (99\%, Alfa Aesar), sodium hydroxide (Sigma Aldrich), tetrahydrofuran (THF, anhydrous, 99.9\%, Sigma Aldrich), triethanolamine (99\%, Sigma Aldrich) and toluene (anhydrous 99.8\%, Sigma Aldrich). Visible light irradiation was performed via two $50 \mathrm{~W}$ LED chips (Foxpic High Power 50 W LED Chip Bulb Light DIY White 3800LM 6500 K) connected to a self-made circuit and cooling system. UV light irradiation was performed via $5 \mathrm{~W}$ LED stripes (Chili-Tec, CLS-100UV) containing 30 UV SMD LEDs (type 5050, $\lambda=395 \mathrm{~nm}$ ). Sonication was performed in a sonication bath from Elma (Transsonic T310). G-CN was synthesized from cyanuric acid-melamine (CM) complex according to the literature and was annotated as CM. ${ }^{39}$

\section{Characterization}

Zeta potential and size (by dynamic light scattering, $\lambda=633 \mathrm{~nm}$ at $\theta=90^{\circ}$, Z-averaged diameters are presented) measurements of colloidal suspensions of CMs were performed with a Zetasizer Nano ZS90 from Malvern. X-ray diffraction (XRD) patterns were obtained using Bruker D8 Advance X-ray diffractometer via $\mathrm{Cu}-\mathrm{K}_{\alpha}$ radiation. Scanning electron microscopy (SEM) was performed using JSM-7500F (JEOL) equipped with an Oxford Instruments X-MAX $80 \mathrm{~mm}^{2}$ detector for the determination of the elemental composition of $\mathrm{CM}$ samples. The structures of materials were also examined by TEM using an EM 912 Omega microscope at 120 
kV. Fourier transform infrared (FT-IR) spectra were acquired on a Nicolet iS 5 FT-IR spectrometer. Solid state ultraviolet-visible (UV-Vis) spectroscopy was recorded via a Cary 500 Scan spectrophotometer equipped with an integrating sphere. Atomic Force Microscopy (AFM) was performed via Digital Instruments by dispersing samples in water and drying on mica. Elemental analyses of the samples were recorded via a Vario Micro device. Photoluminescence spectra of the samples were obtained by a Hitachi F-7000 spectrometer. ${ }^{1} \mathrm{H}$ NMR was recorded at ambient temperature at $400 \mathrm{MHz}$ with a Bruker Ascend400. Sedimentation videos were prepared by taking images of dispersions continuously with a camera from Logitech connected to computer in custom made set up and transformed into a short video.

\section{Time dependent synthesis of AHPA grafted CM}

$50 \mathrm{mg} \mathrm{CM}$ was weighted in a glass vial with magnetic stirrer, $1 \mathrm{~g}$ AHPA solution (40 wt.\% in water) and $1 \mathrm{~g}$ deionized water were added. The mixture was sonicated for 10 minutes and nitrogen was flushed through the mixture for 3 minutes for the removal of dissolved oxygen. The mixture was put between two 50W LED daylight sources (20 $\mathrm{cm}$ apart from each other) and stirred continuously for the desired reaction time. Afterwards, the mixture was vacuum filtered, washed 3 times with water $(3 \times 50 \mathrm{~mL})$ and washed once with acetone $(20 \mathrm{~mL})$. After filtration, the solid sample was dried under vacuum at $60{ }^{\circ} \mathrm{C}$ overnight and the product was obtained in nearly quantitative yield.

\section{Synthesis of 1-decene grafted CM}

$50 \mathrm{mg} \mathrm{CM}$ was weighted in a glass vial with magnetic stirrer, $1 \mathrm{~g}$ 1-decene and $1 \mathrm{~g}$ IPA were added. The mixture was sonicated for 10 minutes and nitrogen was flushed through the mixture for 3 minutes for the removal of dissolved oxygen. The mixture was put between two 50W LED daylight sources (20 cm apart from each other) and stirred continuously for the desired reaction 
times at $50{ }^{\circ} \mathrm{C}$. Afterwards, the mixture was vacuum filtered, washed 3 times with IPA $(3 \times 40$ $\mathrm{mL})$ and washed once with acetone $(20 \mathrm{~mL})$. After filtration, the solid sample was dried under vacuum at $60^{\circ} \mathrm{C}$ overnight and the product was obtained in nearly quantitative yield.

\section{Synthesis of allylamine grafted CM}

$50 \mathrm{mg}$ CM was weighted in a glass vial with magnetic stirrer, $1 \mathrm{~g}$ allylamine and $1 \mathrm{~g}$ ethanol were added. The mixture was sonicated for 10 minutes and nitrogen was flushed through the mixture for 3 minutes for the removal of dissolved oxygen. The mixture was put between two 50W LED daylight sources ( $20 \mathrm{~cm}$ apart from each other) and stirred continuously for 48 hours at $40{ }^{\circ} \mathrm{C}$. After the mixture was taken, it was vacuum filtered, washed 3 times with ethanol $(3 \times 40$ $\mathrm{mL})$ and washed once with acetone $(20 \mathrm{~mL})$. After filtration, the solid sample was dried under vacuum at $60^{\circ} \mathrm{C}$ overnight, and the product was obtained in nearly quantitative yield.

\section{Synthesis of $1 \mathrm{H}, 1 \mathrm{H}, 2 \mathrm{H}$-perfluoro-1-decene grafted CM}

$50 \mathrm{mg} \mathrm{CM}$ was weighted in a glass vial with magnetic stirrer, $1 \mathrm{~g} 1 H, 1 H, 2 H$-perfluoro-1decene and $1 \mathrm{~g}$ THF were added. The mixture was sonicated for 10 minutes and nitrogen was flushed through the mixture for 3 minutes for the removal of dissolved oxygen. The mixture was put between two 50W LED daylight sources $(20 \mathrm{~cm}$ apart from each other) and stirred continuously for 48 hours at $50{ }^{\circ} \mathrm{C}$. After the mixture was taken, it was vacuum filtered and washed 3 times with THF $(3 \times 40 \mathrm{~mL})$ and washed once with acetone $(20 \mathrm{~mL})$. After filtration, the solid sample was dried under vacuum at $60^{\circ} \mathrm{C}$ overnight and the product was obtained in nearly quantitative yield. 


\section{Results and Discussion}

\section{Photoinduced g-CN Functionalization}

To perform photoinduced g-CN functionalizations, allyl containing molecules were chosen as the allyl bond forms stable radicals due to resonance structures. Thus propagation is suppressed, and only single radical additions take place. A one pot visible light induced grafting method was utilized to integrate allyl compounds onto the surface of g-CN, which was synthesized from cyanuric acid-melamine (CM) complex and is annotated as CM. Hydrophilic grafting via AHPA molecule (50 mg CM and 20 wt.\% AHPA solution in water) increases negative charge as well as $\mathrm{S}, \mathrm{O}$ and $\mathrm{Na}$ atoms on the g-CN structure. Table 1 shows the initial analysis results of unmodified and AHPA modified CM by the means of particle size, zeta potential and elemental analysis. Particle size measurements and zeta potential measurements were performed in aqueous dispersion. As grafting time increases particle sizes decrease from $2448 \mathrm{~nm}$ to $1468 \mathrm{~nm}$. However, number averaged diameters show significantly decreased particle sizes as expected (Table S1). As grafting with AHPA introduces additional negative charges to the CN structure increased repelling force between layers might be caused. Therefore decreased particle sizes are observed in the case of CM-AHPA. Grafting onto the surface also causes a spacing effect, which may be another reason for smaller particle size as grafting time increases. Moreover, a significant increase in negative zeta potentials was also observed over the course of the grafting reaction due to grafted sulfonic acid groups from AHPA molecule, while unmodified CM has a zeta potential of $-27.5 \mathrm{mV}$ a change up to $-53.5 \mathrm{mV}$ is observed via increase of negative surface charges. Longer reaction times provide increased grafting densities, as shown by the sulfur content observed via elemental analysis and more stable zeta potentials occurs at the same time (Figure 
1). The relation of reaction time with the size and $\mathrm{C} / \mathrm{N}$ ratio of the modified material can be seen in Figure $\mathrm{S} 1$ where longer grafting times result in lower size and higher $\mathrm{C} / \mathrm{N}$ ratio.

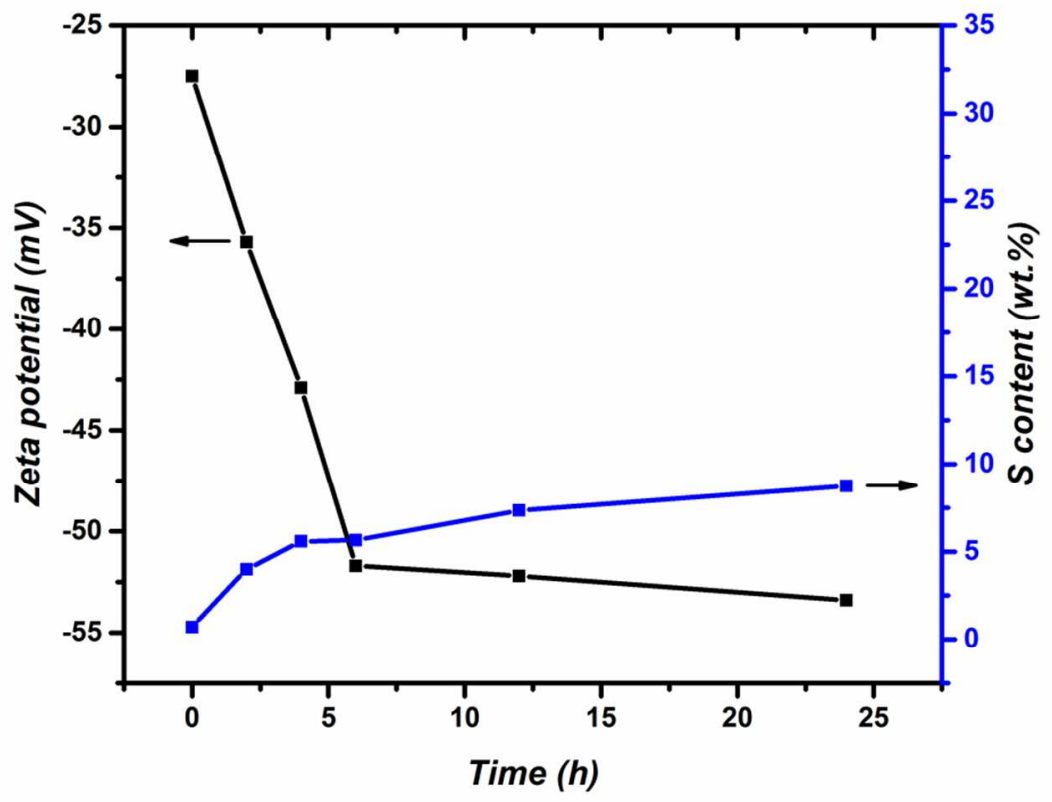

Figure 1. The relation of grafting time with sulfur content and zeta potential of modified CMs.

The double bond activity of AHPA is sufficient to achieve grafting at room temperature via visible light induced grafting, and as can be compared from the sulfur amount, it is possible to increase the sulfur content by a factor of 10 after 24 hours of reaction, namely from $0.7 \%$ up to 8.74 wt.\%. Assuming a sheet model consisting of g-CN sheets with complete grafting of an AHPA layer, a sheet thickness can be estimated from the elemental analysis data acquired from the sample with the highest grafting density. Considering the length of AHPA the maximal overall coating thickness can be estimated to be two times $1.2 \mathrm{~nm}$ for the top and the bottom of the hybrid sheet. The theoretical S weight content for the AHPA layer is $14.7 \mathrm{wt. \%}$. Therefore, the overall hybrid sheet thickness can be estimated to be maximal $4.1 \mathrm{~nm}$ for the grafted structure, which leaves $1.7 \mathrm{~nm}$ for the inner g-CN part. Theoretical assumption calculated via $\mathrm{S}$ 
weight content also fits with the height profiles from AFM results (Figure S2). Thus, this functional delamination can be regarded as very effective.

Table 1. Properties of unmodified and AHPA modified CMs. (reaction parameters: $50 \mathrm{mg}$ CM, $1 \mathrm{~g} 40$ wt.\% AHPA solution in water, $1 \mathrm{~g}$ deionized water, visible light, ambient temperature).

\begin{tabular}{|c|c|c|c|c|c|}
\hline Sample & Time & $D_{\mathrm{n}}(\mathrm{nm})^{\mathrm{a}}$ & Zeta Potential $(\mathrm{mV})$ & S content $(w t . \%)^{b}$ & C/N Ratio ${ }^{b}$ \\
\hline $\mathrm{CM}$ & & 2448 & -27.5 & 0.712 & 0.6025 \\
\hline $\begin{array}{l}\text { CM- } \\
\text { AHPA2 }\end{array}$ & $2 \mathrm{~h}$ & 2105 & -35.7 & 3.997 & 0.8478 \\
\hline $\begin{array}{l}\text { CM- } \\
\text { AHPA4 }\end{array}$ & $4 \mathrm{~h}$ & 1944 & -42.9 & 5.574 & 0.8518 \\
\hline $\begin{array}{l}\text { CM- } \\
\text { AHPA6 }\end{array}$ & $6 \mathrm{~h}$ & 1712 & -51.7 & 5.648 & 0.9125 \\
\hline $\begin{array}{l}\text { CM- } \\
\text { AHPA12 }\end{array}$ & $12 \mathrm{~h}$ & 1610 & -52.2 & 7.345 & 1.0997 \\
\hline $\begin{array}{l}\text { CM- } \\
\text { AHPA24 }\end{array}$ & $24 \mathrm{~h}$ & 1468 & -53.4 & 8.743 & 1.2244 \\
\hline $\begin{array}{l}\text { CM-AHPA } \\
\text { ref. }\end{array}$ & $12 \mathrm{~h}$ & 2278 & -27.6 & 0.697 & 0.6032 \\
\hline
\end{tabular}

In order to exclude physical adsorption of AHPA to the surface, reference experiments were performed. The reference reaction was based on mixing of reactants without visible light irradiation and subsequent purification. After 12 hours the same purification steps were applied as for the irradiated samples and the obtained material was characterized via elemental analysis (Table 1). However no change was observed for the non-irradiated samples, which indicates that 
reaction proceeds on surface of $\mathrm{g}-\mathrm{CN}$ through photoexcitation. The reference experiment suggests that AHPA is grafted to the surface instead of simple physical adsorption. Addition of radical scavenger such as hydroquinone also inhibits the reaction as no change in elemental analysis was observed as shown in Table S2, which indicates a radical mechanism for the grafting reaction. No change was observed in elemental analysis and particle size while mixing just $\mathrm{CM}$ in water under visible light irradiation. AHPA grafted $\mathrm{CM}$ was also dispersed in $\mathrm{D}_{2} \mathrm{O}$ and mixed thoroughly for 2 hours, then filtered, and ${ }^{1} \mathrm{H}-\mathrm{NMR}$ spectra of the solution were taken as shown in Figure S3. Existence of only solvent peak states that the AHPA molecule is grafted to the surface chemically instead of being adsorbed in the pores of $\mathrm{CN}$ and that the applied purification method is sufficient to remove any unreacted starting material. In order to observe the effect of concentration on grafting experiments, variations in allyl compound concentration were conducted as explained in experimental part. Compared to the initial attempted concentration of $20 \mathrm{wt} . \%$ of AHPA, a lower concentration of $10 \mathrm{wt} \%$ as well as an increased concentration of $40 \mathrm{wt} . \%$ was utilized (Table S3). The obtained results match with the results retrieved from time dependent grafting, e.g. smaller sized particles and decrease in zeta potential values are obtained for increased AHPA concentrations. Moreover, less concentrated medium (10 wt.\%) leads to decreased sulfur content compared to higher concentration as expected (3.676 wt.\%). Also, as expected, it is possible to graft increased amounts of AHPA on g-CN with higher concentration of allyl compound (40 wt.\%) as shown by elemental analysis and the increase of the weight percentage of sulfur atom (up to $9.277 \mathrm{wt} . \%$ ).

Electron microscopy techniques were also used to visualize the structures after modification as depicted in Figure S4 and S5. TEM and SEM images show that the material structures are similar before and after grafting while the particle size decreases after grafting reactions. 
Elemental analysis via EDX shows a uniform appearance of $\mathrm{S}, \mathrm{O}$ and $\mathrm{Na}$ atoms throughout the surface of CM-AHPA.

a)

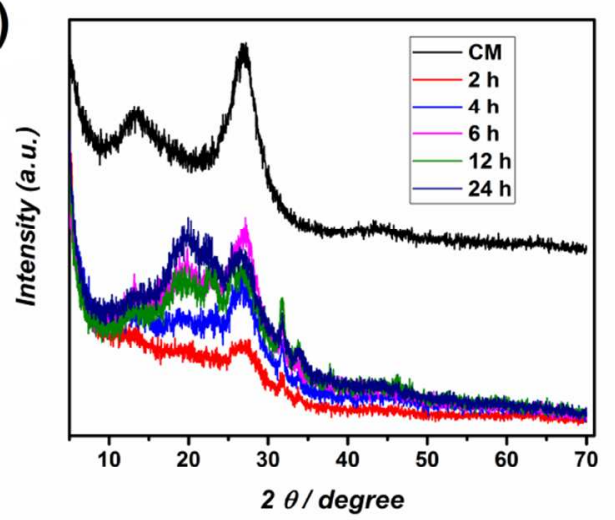

b)

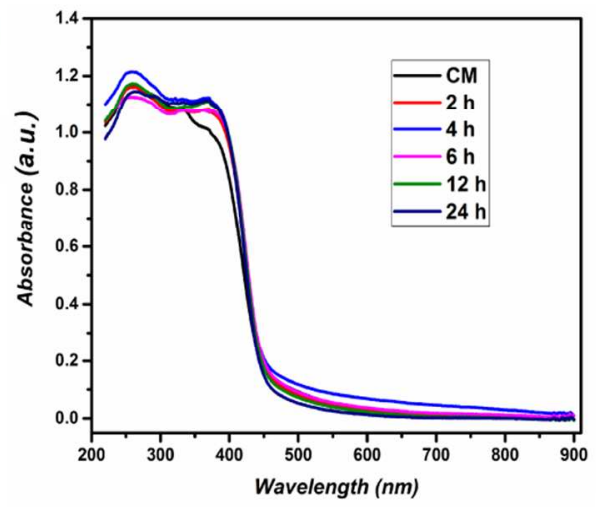

c)
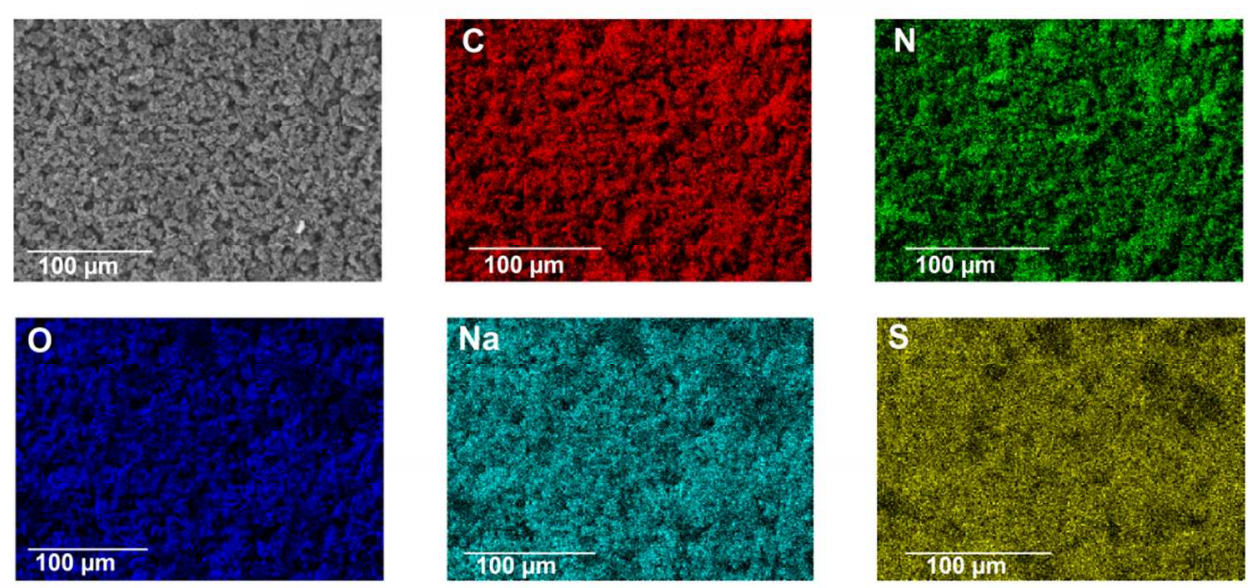

Figure 2. a) XRD profiles of unmodified and AHPA modified CM, b) solid UV-Vis spectra of unmodified and AHPA modified CM and c) elemental mapping of CM-AHPA24 via EDX.

XRD profiles show a change in peak positions (Figure 2a), which may be due to a potential positioning of the positively charged sodium atom between layers. The region between 17-20 degrees can be assigned to the layer-layer stacking and increase in these peaks after modification can be the result of weak scattering due to delamination. Solid UV-Vis spectra follow the same pattern as unmodified CM with a slight increase in the region between 350-430 nm (Figure 2b) 
suggesting preservation of activity towards photocatalytic applications. Certainly, photocatalytic properties depend on layer thickness, g-CN size and surface functionality. Therefore, detailed investigations on photocatalysis have to be performed to in future works. Elemental mapping via EDX of CM-AHPA24 (Figure 2c) indicates the existence of oxygen, sulfur and sodium atoms throughout the surface originating from grafted AHPA molecules. Therefore a homogenous grafting of AHPA on this length scale is to be assumed. FT-IR spectra of CM and AHPA as well as AHPA modified CM (Figure S6) can be utilized to identify the grafted species. After modification, all the dominant peaks of CM are still present, with a slight new peak at $2900 \mathrm{~cm}^{-1}$ and $1050 \mathrm{~cm}^{-1}$, which can be attributed to asymmetric $\mathrm{CH}_{2}$ stretching and sulfoxide bond respectively. Similar results are obtained for concentration dependent experiments as can be seen in Figure S7.

For the introduction of organosoluble moieties, 1-decene was grafted to the surface of CM. Initial experiments were performed at room temperature at a 1-decene concentration of $50 \mathrm{wt} . \%$ in IPA. Even though the dispersion quality in organic solvents increased, changes in the molecular structure could not be proven, which might be due to low double bond activity of 1decene leading to a low grafting density at ambient temperature. To increase the grafting density, experiments were conducted at $50{ }^{\circ} \mathrm{C}$ as delineated in the experimental part. Particle size, zeta potential of elemental analysis of unmodified CM compared to 1-decene modified CM (Table 2) confirm successful grafting under the altered conditions. DLS measurements in acetone dispersion showed decreased particle sizes with increasing reaction times, which can be due to anchoring of long chain molecule to the surface of CM. Zeta potential measurements were performed in acetone dispersion, which is a complicated task. Moreover, the results show only insignificant differences to unmodified CM, which has to be considered carefully. In our opinion 
the observation of only minor changes in zeta potential might be due to the polar aprotic solvent acetone, which is an uncommon solvent for zeta potential measurements due to its poor properties in the stabilization of charged particles. The carbon:nitrogen ratio increases over reaction time, which is an indication of aliphatic grafting. Only small changes in hydrogen weight percentage could be observed (from $1.923 \%$ up to 2.377 wt.\%). The illustrated relation between reaction time, particle size, zeta potential, $\mathrm{H}$ amount and $\mathrm{C} / \mathrm{N}$ ratio can be found in Figure S8.

Table 2. Properties of unmodified and 1-decene modified CMs. (reaction conditions: 50 mg CM, $1 \mathrm{~g}$ 1-decene, $1 \mathrm{~g} \mathrm{IPA}$, visible light, $\left.50^{\circ} \mathrm{C}\right)$.

\begin{tabular}{|c|c|c|c|c|c|}
\hline Sample & Time & $D_{\mathrm{n}}(\mathrm{nm})^{\mathrm{a}}$ & $\begin{array}{l}\text { Zeta Potential } \\
(\mathrm{mV})\end{array}$ & $\begin{array}{l}\mathrm{H} \text { content } \\
(\mathrm{wt} . \%)^{\mathrm{b}}\end{array}$ & C/N Ratio ${ }^{b}$ \\
\hline $\mathrm{CM}$ & & 2581 & -4.6 & 1.923 & 0.6013 \\
\hline CM-Decene2 & $2 \mathrm{~h}$ & 2377 & -5.1 & 2.203 & 0.6431 \\
\hline CM-Decene4 & $4 \mathrm{~h}$ & 2215 & -5.5 & 2.272 & 0.6840 \\
\hline CM-Decene6 & $6 \mathrm{~h}$ & 2098 & -7.0 & 2.345 & 0.7056 \\
\hline CM-Decene12 & $12 \mathrm{~h}$ & 1968 & -7.2 & 2.361 & 0.7372 \\
\hline CM-Decene24 & $24 \mathrm{~h}$ & 1896 & -7.5 & 2.377 & 0.7582 \\
\hline $\begin{array}{l}\text { CM-Decene } \\
\text { ref. }^{\text {c }}\end{array}$ & $12 \mathrm{~h}$ & 2365 & -4.6 & 1.905 & 0.6024 \\
\hline \multicolumn{6}{|c|}{$\begin{array}{l}{ }^{\mathrm{a}} \text { DLS measurements were performed in acetone }(0.05 \mathrm{wt} . \%) \text { and Z-averaged diameters are } \\
\text { presented, }{ }^{\mathrm{b}} \text { obtained via elemental analysis, }{ }^{\mathrm{c}} \text { reference reaction based on mixing reactants } \\
\text { without visible light irradiation and subsequent purification }\end{array}$} \\
\hline
\end{tabular}

XRD profiles of CM and 1-decene modified CMs follow a similar pattern and show only small differences in peak intensities (Figure 3a). The differences in XRD profiles can be attributed to 
slight distortion of the g-CN framework. Moreover, solid UV-Vis spectra of 1-decene modified CM show similar absorption bands as unmodified CM. Therefore, one of the most important features of g-CN, namely light absorption in the visible range, is retained in the modified product (Figure 3b). SEM and TEM images of 1-decene grafted CM show similar structure to CMAHPA and support the assumption that the structure of modified CM is basically unchanged after the grafting reaction (Figure S9). As expected, EDX shows no significant changes as no new elements are introduced. In order to check if there was any physical adsorption, 1-decene modified $\mathrm{CM}$ was put into $\mathrm{CDCl}_{3}$ and mixed for 2 hours. It was then filtered and ${ }^{1} \mathrm{H}-\mathrm{NMR}$ spectrum was taken, which showed only the solvent peak (Figure S10). Moreover, a reference sample was prepared without light irradiation but the same purification, which showed only insignificant differences from untreated CM. Thus, the efficiency of the washing process can be stated as well as physisorption of 1-decene can be excluded.
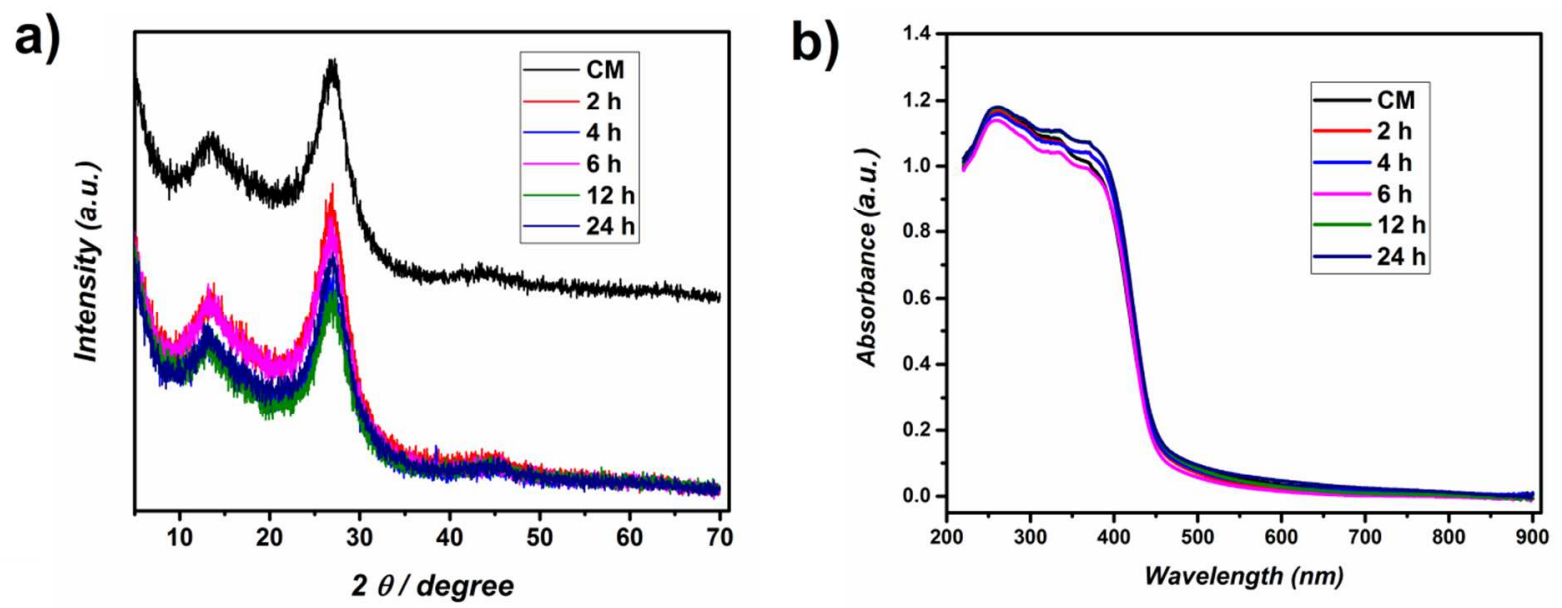

Figure 3. a) XRD and b) solid UV-Vis spectra of unmodified and 1-decene modified CM.

Photoluminescence spectra of CM, AHPA modified CM and 1-decene modified CM follow the exact same pattern, which indicates preservation of $\mathrm{CM}$ photoproperties in the grafted 
products (Figure S11a). Moreover, the appearance of the modified material is very similar to the starting material, where modified samples became a bit paler compared to the unmodified CM (Figure S11b), due the "dilution" with organic compounds.

Mechanistic studies for photodegradation process via $\mathrm{g}-\mathrm{CN}$ have been reported. Irradiation of $\mathrm{g}-\mathrm{CN}$ results in electron excitation and hole formation. Interestingly, either hole oxidation or photoreduction can take place depending on the counter molecule. Methyl orange (MO) degradation via g- $\mathrm{CN}$ is attributed to photoreduction process with a hole oxidation providing a minor contribution where Rhodamine $B(\mathrm{RhB})$ degradation solely conducted via hole oxidation. $^{40,41}$ The mechanism suggested in literature for $\mathrm{g}-\mathrm{CN}$ with double bonds under visible light is based on the theory of formation of $\mathrm{OH}$ radicals and related to valance band values of possible intermediate species. ${ }^{42}$ Regarding the mechanism of grafting, the most probable pathway seems to be radical recombination. Upon irradiation electrons in the valence band of g$\mathrm{CN}$ can be excited leading to an exciton state with adjacent electron hole. Subsequently, the formed radical can react with the respective ene in a radical addition. Finally, the leftover other radical on the surface can recombine with the radical formed at the ene after radical addition, which leads to a new covalent bond between g-CN surface and the ene molecule. Whether the first addition to the ene stems from the electron hole or the excited electron can only be speculated at the current point. As the utilized enes are quite electron-rich it is likely that the first addition originates from the formed electron hole on the g-CN surface, i.e. the grafting agent is first oxidized, then recombines to close the photocatalytic cycle. In order to observe the effects of hole formation and excited electrons on grafting, several control experiments were performed via addition of triethanolamine as hole scavenger or hydrogen peroxide as electron scavenger for AHPA grafting as explained in supporting information. Interestingly, after 4 hours of reaction, 
slight increase in sulfur amounts in the final products can be observed. Yet significantly lower grafting compared with CM-AHPA4 was observed, and no grafting took place when both hydrogen peroxide and triethanolamine were present (Table S4). These results suggest that grafting can also be achieved via only electron hole or excited electron based mechanisms. Nevertheless, significantly lower grafting rate is observed after addition of hole or electron scavengers. Efficient grafting may take place via a process combining of both mechanisms. Hole can abstract an electron from the double bond leaving cation and radical on the molecule that recombines with the electron for grafting. Moreover, hole can react with a water molecule to from a hydroxyl radical that reacts with the double bond. The formed radical can recombine on the surface as well. For the mechanism from excited electron, formation of hydroxyl radical (either from water or oxygen after chain reactions) is an essential step. Hydroxyl radical can perform addition to double bond leaving another radical on the molecule, which can recombine on the surface as well. In a recent article investigating mechanism of water splitting with g-CN it was theoretically shown that water molecule forms a complex with heptazinyl rings of g-CN and after irradiation and abstraction, radical formation takes place on heptazinyl ring and a hydroxyl radical is formed. ${ }^{43}$ Thus, hydroxyl radical could react with double bond a recombination with the heptazinyl radical could take place for grafting.

\section{Dispersion Properties}

Sulfonic acid group is known to be an excellent hydrophilic functional group. ${ }^{44}$ Therefore, the introduction of sulfonic acid functionality to g-CN is expected to result in enhanced dispersibility in water. To test dispersibility of the AHPA modified CM, various conditions were tried. Pure CM precipitates directly from water (Supporting Video 1) and requires long times of sonication 
(as much as 24 hours) for the formation of uniform dispersions. However, CM AHPA is immediately dispersible in water even via gentle shaking by hand (Supporting Video 2). To prepare the $\mathrm{CM}$ dispersion in water, even after 30 minutes of sonication, still most of the $\mathrm{CM}$ is present at the bottom of the vial as depicted in Figure 4a. On the other hand CM-AHPA disperses in water after 5 minutes of sonication (Figure 4b), and the dispersion is uniform and solid particles are not visible in the system. In order to check the stability of water dispersion, the sedimentation of CM and CM-AHPA (1 wt.\% in water, both sonicated for 5 minutes), was investigated. In the case of AHPA modified CM the solid particles are still dispersed in water phase after 3 days (Figure 4c), while CM sediments completely after 8 h (Supporting Video 3).

a)

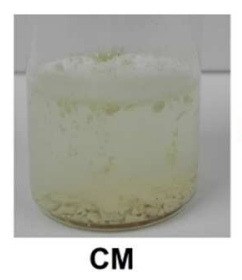

30 mins sonication

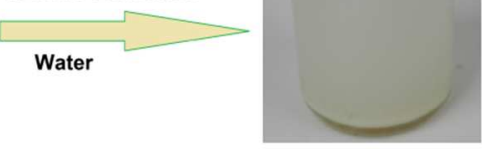

b)

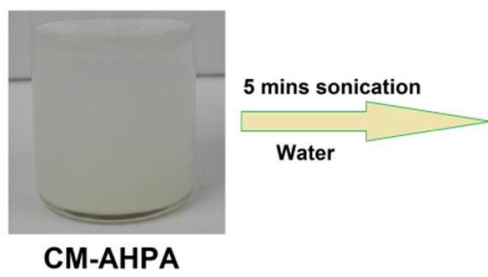

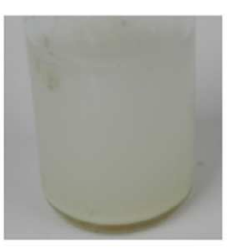

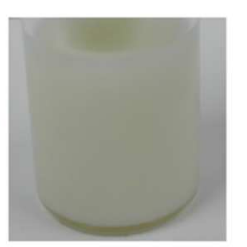

c)

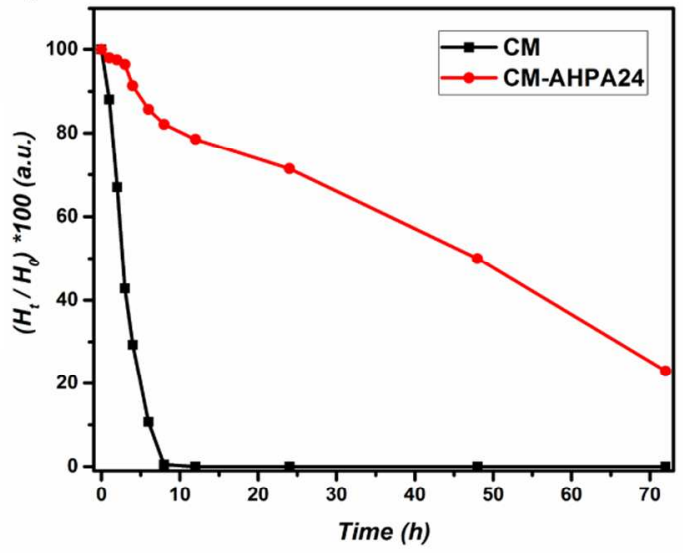

Figure 4. a) $\mathrm{CM}$ in water before and after 30 minutes sonication, b) AHPA modified CM in water before and after 5 minutes sonication, c) sedimentation graph of unmodified and AHPA modified CM in water over 3 days.

To analyze the sedimentation process, initial heights of dispersions $\left(H_{0}\right)$ as well as the heights of the dispersed phases in specific time intervals $\left(H_{\mathrm{t}}\right)$ were measured to yield a sedimentation graph as shown in Figure 4c. Moreover, sedimentation images of CM and AHPA modified CM 
over defined times can be found in Figure S12. All these results confirm the stability of CMAHPA dispersion along with benign dispersion preparation. Dispersions of AHPA modified CM from control experiments with hole or electron scavengers also show stability over 3 hours (Figure S13a), where the product from both electron and hole scavenger reaction has poor dispersibility comparable to non-modified g-CN (Figure S13b). In addition, only small amounts of non-modified g-CNs can be dispersed (as low as 0.05 wt.\%). In contrast, the hydrophilic character of AHPA modified CM also allows to disperse increased amounts of g-CN in water. Therefore, enhanced activity of g-CN might be the case. In order to confirm the stability of high amount CM-AHPA dispersions, 10 wt.\% and 20 wt.\% water dispersions of AHPA modified CM were prepared (Supporting Video 4). The video illustrates that the $20 \%$ solid content system yields really thick dispersion, which prevents sedimentation imaging. Nevertheless, sedimentation set in after 3 hours. In the case of $10 \%$ solid content system, sedimentation was observed gradually over a day while standing. Overall, grafting of sulfonic acid groups onto the surface of g-CN improved the dispersion properties of $\mathrm{g}-\mathrm{CN}$ in water significantly. Dispersions can be prepared in shorter times, are stable over much longer periods, and increased amounts of g-CN can be introduced into dispersion, which is of significant interest for various applications of $\mathrm{g}-\mathrm{CN}$.

a)

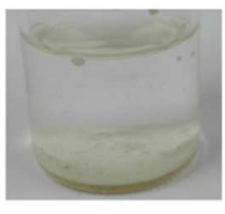

30 mins sonication

Acetone

b)

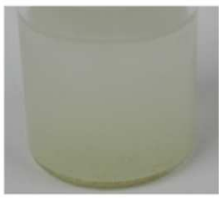

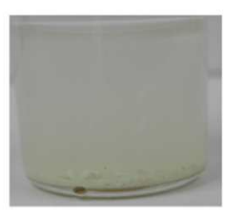

c)

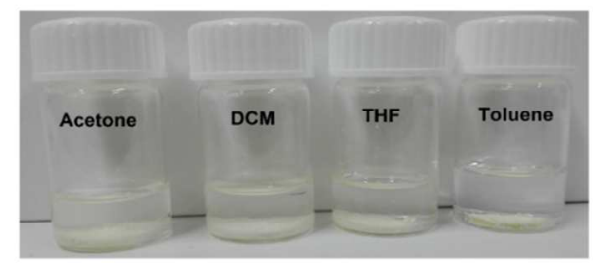

d)

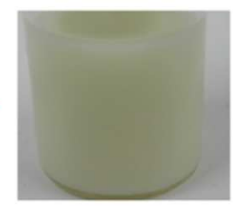


Figure 5. a) $\mathrm{CM}$ in acetone before and after 30 minutes sonication, b) 1-decene modified CM in acetone before and after 5 minutes of sonication, c) 1-decene modified CM in 4 different organic solvents before sonication d) 1-decene modified CM in 4 different organic solvents after 10 minutes sonication $(0.8 \mathrm{wt} . \%)$.

Some applications such as organic coupling require organic solvent as medium. However, g$\mathrm{CN}$ entails usually poor organic dispersibility. Therefore, the medium chain alkene 1-decene was used to improve the dispersion in organic media. In the case of $\mathrm{CM}$, only poor dispersibility in organic solvents exists, e.g. in acetone before and after 30 minutes of sonication, where most of the material remains non-dispersed (Figure 5a). On the other hand 1-decene modified CM yields good dispersions in acetone after 5 minutes sonication (Figure $5 b$ ). The stability of acetone dispersions (1 wt.\%) was assessed via sedimentation (Supporting Video 5). The 1-decene modified CM stays dispersed over a significantly longer period than unmodified CM (Figure S14). The images of unmodified and 1-decene modified CM dispersions in acetone and their sedimentation over distinct periods confirm the partial stability of 1-decene modified CM dispersions in acetone. Other common organic solvents were also investigated as shown in Figure 5c-d. The addition of 1-decene modified CM to acetone, DCM, THF and toluene leads in all the examined cases to uniform dispersions after 10 minutes of sonication Moreover, it could be shown that the solid content of CM-decene could be increased up to 2 wt.\% in organic dispersions. Overall, grafting with 1-decene enhances the organic character of g-CN which is beneficial for the preparation of organic dispersions and might be useful for a variety of applications. 


\section{Introduction of Functionalities}

In order to show the generality of the approach, functionalities were introduced as well, namely amine and fluoro functionalities via allylamine and perfluoro-1-decene, respectively. Possessing an amino group, allylamine can be protonated in acidic conditions, which leads to cationic, but $\mathrm{pH}$ sensitive materials. On the other hand fluorinated 1-decene might lead to fluorophilic materials. In addition, the fluorinated molecule allows easy detection of grafting as fluorine is a marker atom. Reactions were performed as explained in experimental part in a one pot procedure under visible light. Allylamine grafting was achieved in ethanol at $40{ }^{\circ} \mathrm{C}$, and fluoro-modification was performed in $\mathrm{THF}$ at $50{ }^{\circ} \mathrm{C}$. Initial results did not show a significant size change or altered zeta potential values (Table 3): However, increased $\mathrm{C} / \mathrm{N}$ ratios were observed in both cases. Since g-CN has better absorption in the UV range; grafting with 1-decene or perfluoro-1-decene was also investigated via UV light irradiation at a wavelength of $395 \mathrm{~nm}$. It was concluded that grafting via UV light is indeed more effective with enes that show otherwise low activity, which is in line with the already discussed recombination mechanism that is most likely the base for the grafting reaction. In the case of UV light induced grafting, also lower reaction temperatures can be utilized (Table S5).

Table 3. Properties of unmodified and allylamine and perfluoro decene modified CMs.

\begin{tabular}{|l|l|l|l|}
\hline Sample & $D_{\mathrm{n}}(\mathrm{nm})^{\mathrm{a}}$ & Zeta Potential $(\mathrm{mV})$ & C/N Ratio \\
\hline CM & 2448 & -27.5 & 0.6025 \\
\hline CM-AA & 2265 & -28.9 & 0.7433 \\
\hline
\end{tabular}




\begin{tabular}{|l|l|l|l|}
\hline CM-F & 2198 & -6.4 (in acetone) & 0.6617 \\
\hline CM-AA reference $^{c}$ & 2376 & -26.9 & 0.6112 \\
\hline CM-F reference $^{c}$ & 2407 & -4.7 (in acetone) & 0.6054 \\
\hline
\end{tabular}

${ }^{\mathrm{a}}$ DLS measurements were performed in acetone (0.05 wt.\%) and Z-averaged diameters are presented, ${ }^{\mathrm{b}}$ obtained by elemental analysis results, ${ }^{\mathrm{c}}$ reference reaction based on mixing reactants without visible light irradiation and subsequent purification

SEM images of CM-AA and CM-F materials again did not show any significant structural differences on the particle level (Figure S15 a and b). Elemental mapping of perfluoro decene grafted CM clearly indicates the existence of fluorine atoms over the surface (Figure 6a and Figure S15c).

a)
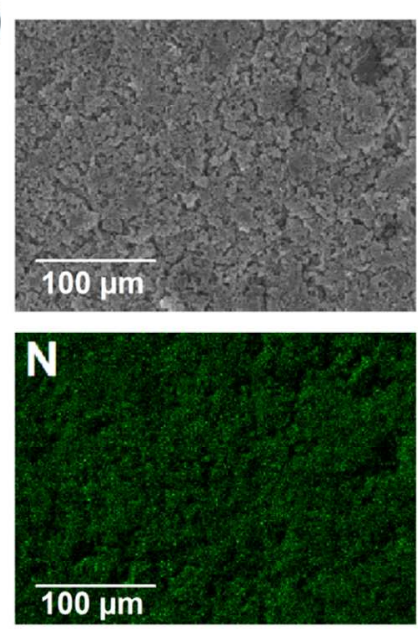
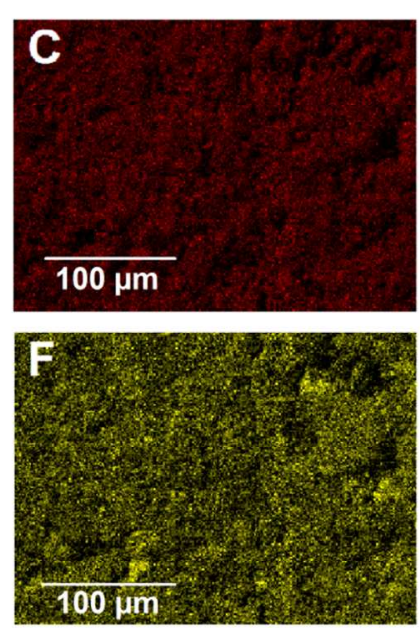

b)

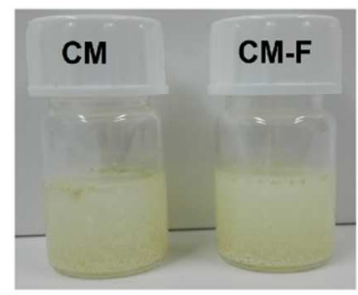

c)

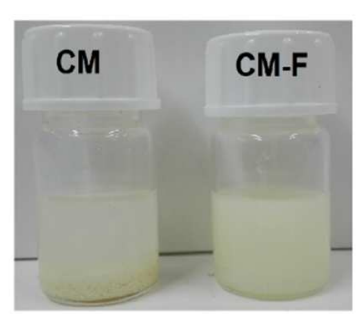

Figure 6. a) Elemental mapping of perfluoro decene grafted CM via EDX, b) $\mathrm{CM}$ and fluoro modified $\mathrm{CM}$ (CM-F) in hexafluorobenzene before sonication, c) $\mathrm{CM}$ and $\mathrm{CM}-\mathrm{F}$ in hexafluorobenzene after 30 minutes sonication. 
Introduction of fluorine atoms provides fluorophilic character to g-CN, which allows dispersibility in fluorosolvents. For example hexafluorobenzene was used as solvent and 0.4 wt.\% non-modified and fluoro modified CM were dispersed (Figure 6b-c). Sonication for 30 minutes does not lead to dispersion of non-modified $\mathrm{CM}$ as almost all of the material remains non-dispersed at the bottom of the vial. A similar result was observed when CM-decene was utilized in hexafluorobenzene (Figure S16). On the other hand CM-F has better dispersion properties and yields a uniform dispersion. However it is important to note that sedimentation of this dispersion starts after 5 minutes and sediments completely after 1 hour. Theoretically the increased density of hexafluorobenzene should slow sedimentation down. Nevertheless, fast sedimentation was observed that might be due to the low dielectric constant of the solvent hexafluorobenzene, which promotes Hamaker forces between the sheets. Yet, it is easy to redisperse the material even via gentle shaking by hand. Therefore the fluoro grafted material can be of great interest as with a catalyst within fluorinated solvents under continuous mixing.

\section{pH Dependent Dispersion}

Allylamine functionality was introduced to illustrate the option of a $\mathrm{pH}$ dependent dispersibility in aqueous media. In acidic $\mathrm{pH}(\mathrm{pH}=4)$, allylamine modified $\mathrm{CM}$ yields uniform dispersions via protonation of amino groups. On the other hand increasing the $\mathrm{pH}$ of the medium to the basic range $(\mathrm{pH}=9)$ causes immediate precipitation of solid particles due to deprotonation of amino groups (Figure 7). Re-acidification of the medium leads to uniform dispersions once again, so the dispersion process is reversible. Addition of acid to a basic dispersion of allylamine modified CM (1 wt.\%) can be found in Supporting Video 6. In aqueous media, allylamine modified CM shows high $\mathrm{pH}$ sensitivity and can be effective when acidic conditions are needed. 
Moreover, ease of separation after base addition makes this material highly beneficial for $\mathrm{pH}$ dependent reaction media and recycling of the catalytic material. Another attractive application that might be accessible via ionically modified $\mathrm{CM}$ is anion-cation-driven layer-by-layer deposition, which is a method that generates well defined superstructures on surfaces and in dispersion. ${ }^{45,46}$
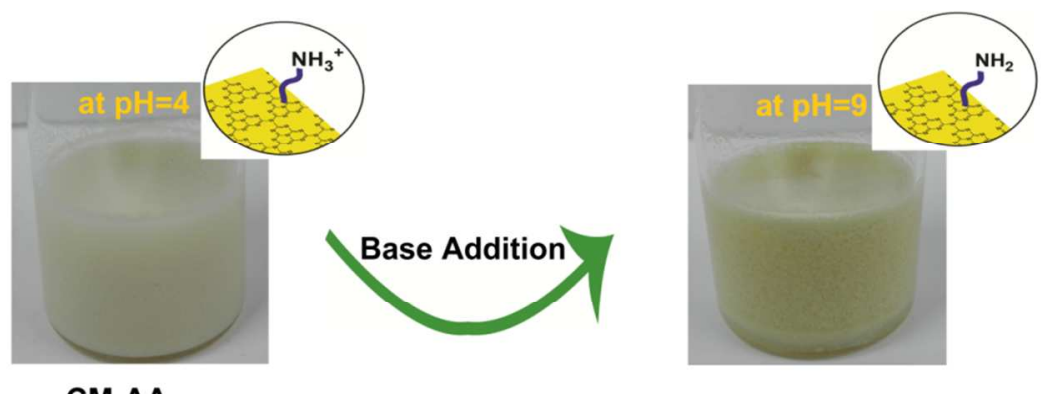

CM-AA

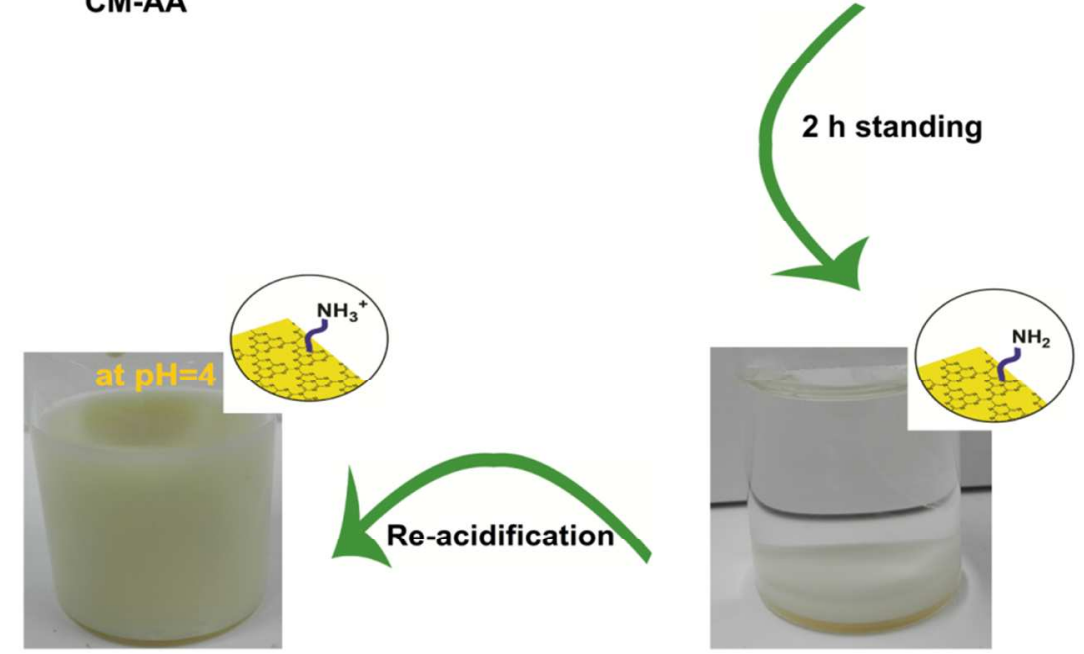

Figure 7. Allylamine modified $\mathrm{CM}$ in water $(1 \mathrm{wt} . \%)$ at acidic $\mathrm{pH}(\mathrm{pH}=4)$, observation of immediate precipitation after base addition $(\mathrm{pH}=9)$, complete sedimentation after standing for 2 hours and redispersion after re-acidification $(\mathrm{pH}=4)$.

\section{Conclusion}


Overall, we introduced visible light induced, one pot grafting approach for $\mathrm{g}-\mathrm{CN}$ for enhanced dispersion features, while keeping the photoproperties unchanged. Using molecules with allylic double bonds avoids monomer polymerization and provides a monolayer dispersion stabilization. Sulfonic acid group of AHPA provides excellent hydrophilicity and grafting 1-decene provides significant organo dispersibility for $\mathrm{g}$-CN. These changes result in minimal times for redispersion preparation, higher dispersion stability and increasing the possible highest solid content (e.g. or hybrid formation) of dispersed systems significantly. It is also possible to introduce $\mathrm{pH}$ dependent dispersion stability on $\mathrm{CM}$ via allylamine groups or fluorophilic character by integrating perfluoro-1-decene molecule. To the best of our knowledge, this research is the most detailed approach for the enhanced dispersibility of g-CNs in different media. The presented method can help to expand the whole g-CN field as dispersibility is a significant aspect for heterogeneous catalysis systems.

Supporting Information. Additional synthetic procedures, analytical data and video files are collated in Supporting Information. This material is available free of charge via the Internet at http://pubs.acs.org.

\section{AUTHOR INFORMATION}

\section{Corresponding Author}

*(BVKJS) Tel (+49) 331567 9509; Fax (+49) 331567 9502; Email

bernhard.schmidt@mpikg.mpg.de

\section{Author Contributions}


The manuscript was written through contributions of all authors. All authors have given approval to the final version of the manuscript.

\section{Funding Sources}

Max-Planck society

\section{ACKNOWLEDGMENT}

The authors thank the Max Planck Society for financial support, Mrs. Heike Runge for the SEM and EDX measurements, Ms. Rona Pitschke for TEM measurements, Ms. Sylvia Ost for elemental analysis, Ms. Anneliese Heilig for AFM measurements, Dr. Aleksandr Savateev for PL measurements and Dr. Klaus Tauer for the access of custom made set up for observing sedimentations.

\section{REFERENCES}

(1) Zheng, Q.; Durkin, D. P.; Elenewski, J. E.; Sun, Y.; Banek, N. A.; Hua, L.; Chen, H.; Wagner, M. J.; Zhang, W.; Shuai, D. Visible-Light-Responsive Graphitic Carbon Nitride: Rational Design and Photocatalytic Applications for Water Treatment. Environ. Sci. Technol. 2016, 50, 12938-12948.

(2) Schwinghammer, K.; Tuffy, B.; Mesch, M. B.; Wirnhier, E.; Martineau, C.; Taulelle, F.; Schnick, W.; Senker, J.; Lotsch, B. V. Triazine-based carbon nitrides for visiblelight-driven hydrogen evolution. Angew. Chem., Int. Ed. Engl. 2013, 52, 2435-2439.

(3) Dong, G.; Zhang, Y.; Pan, Q.; Qiu, J. A fantastic graphitic carbon nitride (gC3N4) material: Electronic structure, photocatalytic and photoelectronic properties. J. Photoc. Photobio. C 2014, 20, 33-50.

(4) Gu, Q.; Gao, Z.; Zhao, H.; Lou, Z.; Liao, Y.; Xue, C. Temperature-controlled morphology evolution of graphitic carbon nitride nanostructures and their photocatalytic activities under visible light. $R S C A d v$. 2015, 5, 49317-49325.

(5) Xu, J.; Li, Y.; Peng, S.; Lu, G.; Li, S. Eosin Y-sensitized graphitic carbon nitride fabricated by heating urea for visible light photocatalytic hydrogen evolution: the effect of the pyrolysis temperature of urea. Phys. Chem. Chem. Phys. 2013, 15, 7657-7665. 
(6) Zheng, Y.; Zhang, Z.; Li, C. A comparison of graphitic carbon nitrides synthesized from different precursors through pyrolysis. J. Photoc. Photobio. A 2017, 332, 3244.

(7) Cui, Q.; Xu, J.; Wang, X.; Li, L.; Antonietti, M.; Shalom, M. Phenyl-Modified Carbon Nitride Quantum Dots with Distinct Photoluminescence Behavior. Angew. Chem., Int. Ed. Engl. 2016, 55.

(8) Lin, J.; Pan, Z.; Wang, X. Photochemical Reduction of CO2by Graphitic Carbon Nitride Polymers. ACS Sustain Chem. Eng. 2014, 2, 353-358.

(9) Butchosa, C.; Guiglion, P.; Zwijnenburg, M. A. Carbon Nitride Photocatalysts for Water Splitting: A Computational Perspective. J. Phys. Chem. C 2014, 118, 24833-24842.

(10) Martin, D. J.; Qiu, K.; Shevlin, S. A.; Handoko, A. D.; Chen, X.; Guo, Z.; Tang, J. Highly Efficient Photocatalytic H2 Evolution from Water using Visible Light and StructureControlled Graphitic Carbon Nitride Angew. Chem., Int. Ed. Engl. 2014, 53.

(11) Wang, X.; Maeda, K.; Thomas, A.; Takanabe, K.; Xin, G.; Carlsson, J. M.; Domen, K.; Antonietti, M. A metal-free polymeric photocatalyst for hydrogen production from water under visible light. Nat. Mater. 2009, 8, 76-80.

(12) Zhao, Y.; Shalom, M.; Antonietti, M. Visible light-driven graphitic carbon nitride (g-C3N4) photocatalyzed ketalization reaction in methanol with methylviologen as efficient electron mediator. Appl. Catal. B-Environ. 2017.

(13) Ai, B.; Duan, X.; Sun, H.; Qiub, X.; Wang, S. Metal-free graphene-carbon nitride hybrids for photodegradation of organic pollutants in water. Catal. Today 2015, 258.

(14) Liu, S.; Sun, H.; Ang, H. M.; Tade, M. O.; Wang, S. Integrated oxygen-doping and dye sensitization of graphitic carbon nitride for enhanced visible light photodegradation. $J$. Colloid Interface Sci. 2016, 476, 193-199.

(15) Xu, J.; Antonietti, M. The Performance of Nanoparticulate Graphitic Carbon Nitride as an

Amphiphile. J. Am. Chem. Soc. 2017.

(16) Zheng, Y.; Jiao, Y.; Qiao, S. Z. Engineering of Carbon-Based Electrocatalysts for Emerging Energy Conversion: From Fundamentality to Functionality. Adv. Mater. 2015, 27 , 5372-5378.

(17) Kiskan, B.; Zhang, J.; Wang, X.; Antonietti, M.; Yagci, Y. Mesoporous Graphitic Carbon Nitride as a Heterogeneous Visible Light Photoinitiator for Radical Polymerization. ACS Macro Lett. 2012, 1, 546-549.

(18) Poostforooshan, J.; Badiei, A.; Kolahdouz, M.; Weber, A. P. Synthesis of Spherical Carbon Nitride-Based Polymer Composites by Continuous AerosolPhotopolymerization with Efficient Light Harvesting. ACS Appl. Mater. Inter. 2016, 8, 2173121741.

(19) Kumru, B.; Shalom, M.; Antonietti, M.; Schmidt, B. V. K. J. Reinforced Hydrogels via Carbon Nitride Initiated Polymerization. Macromolecules 2017, 50, 1862-1869.

(20) Sun, J.; Schmidt, B. V.; Wang, X.; Shalom, M. Self-Standing Carbon NitrideBased Hydrogels with High Photocatalytic Activity. ACS Appl. Mater. Inter. 2017, 9, 2029 2034.

(21) Striegler, K.: Modified graphitic carbon nitrides for photocatalytic hydrogen evolution from water: copolymers; Springer, 2015. 
(22) Ishida, Y.; Chabanne, L.; Antonietti, M.; Shalom, M. Morphology control and photocatalysis enhancement by the one-pot synthesis of carbon nitride from preorganized hydrogen-bonded supramolecular precursors. Langmuir 2014, 30, 447-451.

(23) Wan, S.; Zhong, Q.; Ou, M.; Zhang, S.; Cai, W. Facial synthesis of sheet-like carbon nitride from preorganized hydrogen bonded supramolecular precursors and its high efficient photocatalytic oxidation of gas-phase NO. J. Photoc. Photobio. A 2017, 340, 136-145.

(24) Wang, J.; Zhang, L.; Long, F.; Wang, W.; Gu, Y.; Mo, S.; Zou, Z.; Fu, Z. Solvent-free catalytic synthesis and optical properties of super-hard phase ultrafine carbon nitride nanowires with abundant surface active sites. RSC Adv. 2016, 6, 23272-23278.

(25) Zhang, H.; Yu, A. Photophysics and Photocatalysis of Carbon Nitride Synthesized at Different Temperatures. J. Phys. Chem. C 2014, 118, 11628-11635.

(26) Li, H.; Wang, L.; Liu, Y.; Lei, J.; Zhang, J. Mesoporous graphitic carbon nitride materials: synthesis and modifications. Res. Chem. Intermediat. 2015, 42, 3979-3998.

(27) Zheng, Y.; Jiao, Y.; Chen, J.; Liu, J.; Liang, J.; Du, A.; Zhang, W.; Zhu, Z.; Smith, S. C.; Jaroniec, M.; Lu, G. Q.; Qiao, S. Z. Nanoporous graphitic-C3N4@carbon metalfree electrocatalysts for highly efficient oxygen reduction. J. Am. Chem. Soc. 2011, 133, 2011620119.

(28) Daems, N.; Sheng, X.; Vankelecom, I. F. J.; Pescarmona, P. P. Metal-free doped carbon materials as electrocatalysts for the oxygen reduction reaction. J. Mater. Chem. A 2014, 2 .

(29) Zhang, Y.; Zhang, Q.; Shi, Q.; Cai, Z.; Yang, Z. Acid-treated g-C3N4 with improved photocatalytic performance in the reduction of aqueous $\mathrm{Cr}$ (VI) under visible-light. Sep. Purif. Technol. 2015, 142, 251-257.

(30) Yang, S.; Zhou, W.; Ge, C.; Liu, X.; Fang, Y.; Li, Z. Mesoporous polymeric semiconductor materials of graphitic-C3N4: general and efficient synthesis and their integration with synergistic AgBr NPs for enhanced photocatalytic performances. $R S C A d v$. 2013, 3, 5631 .

(31) Wang, H.; Yuan, X.; Wu, Y.; Zeng, G.; Chen, X.; Leng, L.; Li, H. Synthesis and applications of novel graphitic carbon nitride/metal-organic frameworks mesoporous photocatalyst for dyes removal. Appl. Catal. B-Environ. 2015, 174-175, 445-454.

(32) Schlogl, R. Heterogeneous catalysis. Angew. Chem., Int. Ed. Engl. 2015, 54, 3465-3520.

(33) Zheng, Y.; Zhang, Z.; Li, C.; Proulx, S. Surface hydroxylation of graphitic carbon nitride: Enhanced visible light photocatalytic activity. Mater. Res. Bull. 2016, 84, 46-56.

(34) Baig, R. B.; Verma, S.; Nadagouda, M. N.; Varma, R. S. Room temperature synthesis of biodiesel using sulfonated graphitic carbon nitride. Sci. Rep. 2016, 6, 39387.

(35) Gillan, E. G. Synthesis of Nitrogen-Rich Carbon Nitride Networks from an Energetic Molecular Azide Precursor. Chem. Mater. 2000, 12.

(36) Reddy, A. L. M.; Srivastava, A.; Gowda, S. R.; Gullapalli, H.; Dubey, M.; Ajayan, P. M. Synthesis of Nitrogen-Doped Graphene Films For Lithium Battery Application. ACS Nano 2010, 4.

(37) Han, Q.; Wang, B.; Zhao, Y.; Hu, C.; Qu, L. A Graphitic-C3N4 "Seaweed" Architecture for Enhanced Hydrogen Evolution. Angew. Chem., Int. Ed. Engl. 2015, 54, 1143311437.

(38) Bu, X.; Li, J.; Yang, S.; Sun, J.; Deng, Y.; Yang, Y.; Wang, G.; Peng, Z.; He, P.; Wang, X.; Ding, G.; Yang, J.; Xie, X. Surface Modification of C3N4 through Oxygen-Plasma 
Treatment: A Simple Way toward Excellent Hydrophilicity. ACS Appl. Mater. Inter. 2016, 8, 31419-31425.

(39) Shalom, M.; Inal, S.; Fettkenhauer, C.; Neher, D.; Antonietti, M. Improving carbon nitride photocatalysis by supramolecular preorganization of monomers. J. Am. Chem. Soc. 2013, 135, 7118-7121.

(40) Yan, S. C.; Li, Z. S.; Zou, Z. G. Photodegradation performance of g-C3N4 fabricated by directly heating melamine. Langmuir 2009, 25, 10397-10401.

(41) Yan, S. C.; Li, Z. S.; Zou, Z. G. Photodegradation of rhodamine B and methyl orange over boron-doped g-C3N4 under visible light irradiation. Langmuir 2010, 26, 3894-3901.

(42) Liu, J.; An, T.; Chen, Z.; Wang, Z.; Zhou, H.; Fan, T.; Zhang, D.; Antonietti, M. Carbon nitride nanosheets as visible light photocatalytic initiators and crosslinkers for hydrogels with thermoresponsive turbidity. J. Mater. Chem. A 2017, 5, 8933-8938.

(43) Ehrmaier, J.; Karsili, T. N. V.; Sobolewski, A. L.; Domcke, W. Mechanism of Photocatalytic Water Splitting with Graphitic Carbon Nitride: Photochemistry of the HeptazineWater Complex. J. Phys. Chem. A 2017, 121, 4754-4764.

(44) Ayyaru, S.; Ahn, Y.-H. Application of sulfonic acid group functionalized graphene oxide to improve hydrophilicity, permeability, and antifouling of PVDF nanocomposite ultrafiltration membranes. J. Membrane Sci. 2017, 525, 210-219.

(45) Kovtyukhova, N. I.; Ollivier, P. J.; Martin, B. R.; Mallouk, T. E.; Chizhik, S. A.; Buzaneva, E. V.; Gorchinskiy, A. D. Layer-by-Layer Assembly of Ultrathin Composite Films from Micron-Sized Graphite Oxide Sheets and Polycations. Chem. Mater. 1999, 11, 771-778.

(46) Lvov, Y.; Decher, G.; Mohwald, H. Assembly, Structural Characterization, and Thermal Behavior of Layer-by-Layer Deposited Ultrathin Films of Poly(viny1 sulfate) and Poly(allylamine). Langmuir 1993, 9, 481-486. 